\title{
Cell Growth Stimulation, Cell Cycle Alternation, and Anti-Apoptosis Effects of Bovine Bone Collagen Hydrolysates Derived Peptides on MC3T3-E1 Cells Ex Vivo
}

\author{
Jianing Wang ${ }^{1,2}$, Junli Liu ${ }^{1}$ and Yanchuan Guo ${ }^{1,2, *}$ \\ 1 Key Laboratory of Photochemical Conversion and Optoelectronic Materials, \\ Technical Institute of Physics and Chemistry, Chinese Academy of Sciences, Beijing 100190, China; \\ wangjianing@mail.ipc.ac.cn (J.W.); junliliu306@163.com (J.L.) \\ 2 School of Chemical Sciences, University of Chinese Academy of Sciences, Yuquan Road 19A, \\ Beijing 100049, China \\ * Correspondence: yanchuanguo@mail.ipc.ac.cn; Tel.: +86-10-82543583
}

Academic Editor: Toru Ogasawara

Received: 26 March 2020; Accepted: 12 May 2020; Published: 14 May 2020

\begin{abstract}
Bovine bone collagen hydrolysates promote bone formation through regulating bone growth However, the peptide sequences within these isolates have not been characterized. In this study, twenty-nine peptides from bovine bone collagen hydrolysates were purified and identified using nano-HPLC-MS-MS and Peak Studio analysis. HHGDQGAPGAVGPAGPRGPAGPSGPAGKDGR (Deamidation) and GPAGANGDRGEAGPAGPAGPAGPR (Deamidation) enhanced cell viability, inhibited apoptosis, and significantly altered the cell cycle of MC3T3-E1 osteoblast cells. These peptides were selected to perform molecular docking analysis to examine the mechanism underlying these bioactivities. Molecular docking analysis showed that these two peptides formed hydrophobic interactions and hydrogen bonds with epidermal growth factor receptor (EGFR) to activate the EGFR-signaling pathway, which may explain their bioactivity. These findings indicate that these and other similar peptides might be candidates for the treatment of osteoporosis.
\end{abstract}

Keywords: bovine bonecollagen hydrolysates; EGFR; MC3T3-E1 cells; apoptosis; proliferation; cell cycle distribution

\section{Introduction}

Osteoporosis, a common disorder, occurs as a result of unbalanced osteoblastic bone formation and osteoclastic bone resorption [1]. Osteoporosis has influenced the lives of more than 200 million people [2]. Current treatments include medicines, including fluoride [3], that promote bone formation or those, such as bisphosphonates [4], that arrest bone resorption. These pharmaceuticals may cause several side effects including nausea and abdominal pain [5]. Therefore, there is a need for safer osteoporosis treatments with fewer side effects.

Collagen exists in skin, bones, and connective tissue and can be obtained from different sources including both marine and terrestrial animals. However, most collagen is currently obtained from pork skin and bovine bones. Moreover, bovine collagen is used in many applications in the food and cosmetics industries [6,7].

Collagen hydrolysates are usually obtained by enzymatic hydrolysis of collagen. Collagen hydrolysates could provide benefits for the skeleton [8]. Collagen hydrolysates used as food supplements improve the compositional and biodynamic characteristics of vertebrae in ovariectomized rats [9]. Moreover, collagen hydrolysates appear to be more effective than collagen. The vertebrae of ovariectomized rats 
fed a collagen hydrolysate withstood a load four times that withstood by those fed with collagen [9]. Therefore, in this study, collagen hydrolysates were subjected to further study.

Previously, we showed that bovine bone collagen hydrolysates can improve osteoporosis at cellular, animal, and clinical levels. Bovine bone collagen hydrolysates can promote the proliferation and differentiation of osteoblasts [10]. Additionally, bone loss in ovariectomized rats is inhibited by oral administration of bovine bone collagen hydrolysates [11]. Ovariectomized rats are common models to study the effects of collagen hydrolyates on bone loss. Moreover, clinical study results indicate that administration of collagen peptides combined with Caltrate D can promote bone formation [12]. However, the constituents contributing to the bioactivity of these peptides, as well as the underlying mechanisms responsible for their action, remain unknown. Therefore, we isolated the collagen peptide fraction, which increased proliferation and stimulation activity in osteoblasts, and used nano-HPLC-MS-MS (nano-High Performance Liquid Chromatography-Mass Spectrometry-Mass Spectrometry) and Peak Studio software to identify the bioactive peptides. Subsequently, molecular docking analysis using the identified peptides and epidermal growth factor receptor (EGFR) was performed to screen and clarify the underlying mechanisms through which these osteogenic peptides act. The peptides with greater affinity for EGFR were synthesized and their bioactivity was verified using 3-(4,5-Dimethylthiazol-2-yl)-2,5-diphenyltetrazolium bromide (MTT), CCK8(Cell Counting Kit-8), cell cycle analysis, and apoptosis assays. Finally, to identify the underlying mechanisms of action, the affinity between EGFR and EGF (epidermal growth factor) was compared to that between EGFR and the peptides with the greatest bioactivity.

\section{Results}

\subsection{Bovine Bone Collagen Hydrolysate (BBCH) Fractionation via Ultrafiltration}

$\mathrm{BBCH}$ was fractioned into two fractions, BBCH-1 (MW < $3000 \mathrm{Da})$ and BBCH-2 (MW > $3000 \mathrm{Da})$, by ultrafiltration. Stimulation of osteoblast proliferation by these two fractions was evaluated using the IncuCyte system [13]. BBCH-1 (0.05 mg/mL) promoted proliferation of MC3T3-E1 osteoblasts within $72 \mathrm{~h}$ (Figure 1a). Thus, BBCH-1 was subjected to further investigation.

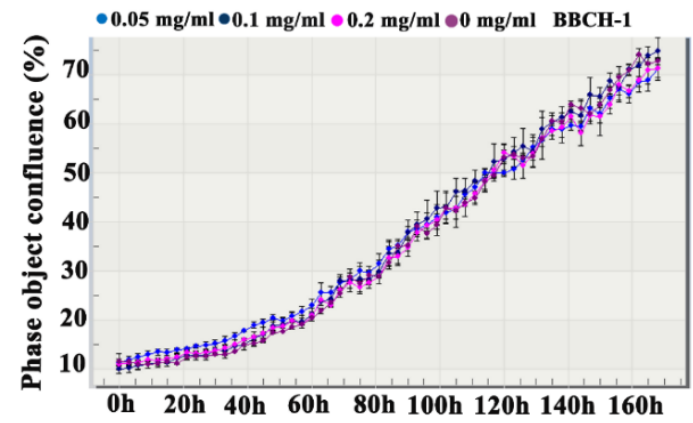

(a)

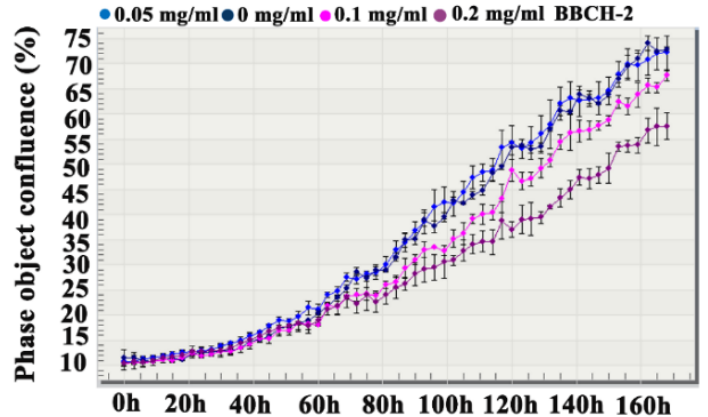

(b)

Figure 1. BBCH-1 and BBCH-2 fractions stimulated the proliferation of MC3T3-E1 cells. (a,b) MC3T3-E1 growth curves after treatment with $\mathrm{BBCH}-1$ and $\mathrm{BBCH}-2$, respectively. The y-axis is a label-free measure of cell confluence utilized by IncuCyte ZOOM live cell imaging system to evaluate cell proliferation. $\mathrm{BBCH}$, bovine bone collagen hydrolysate.

\subsection{Identification of Peptides in the BBCH-1 Fraction}

The sequences of $\mathrm{BBCH}-1$ fraction peptides that had greater osteoblast proliferation stimulation activity were identified using nano-HPLC-MS-MS BBCH-1 (Waters, Milford, MA, USA) analysis and a Bos Taurus databasedriven search via PEAKS Studio 6.0 software (Bioinformatics Solution Inc., Waterloo, ON, Canada). Twenty-nine peptides were identified as were their calculated molecular masses, peptide sequences, and additional information (Table 1). The identified peptide length ranged 
from 10 to 31 amino acids and their corresponding calculated molecular masses ranged from 967.4545 to 2785.3391 Da. Moreover, 27 of the identified peptides were derived from collagen type I and only two were derived from collagen type $X$.

Table 1. Molecular peptide profiles from the bovine bone collagen hydrolysate (BBCH)-1 fraction as identified by nano-HPLC-MS-MS.

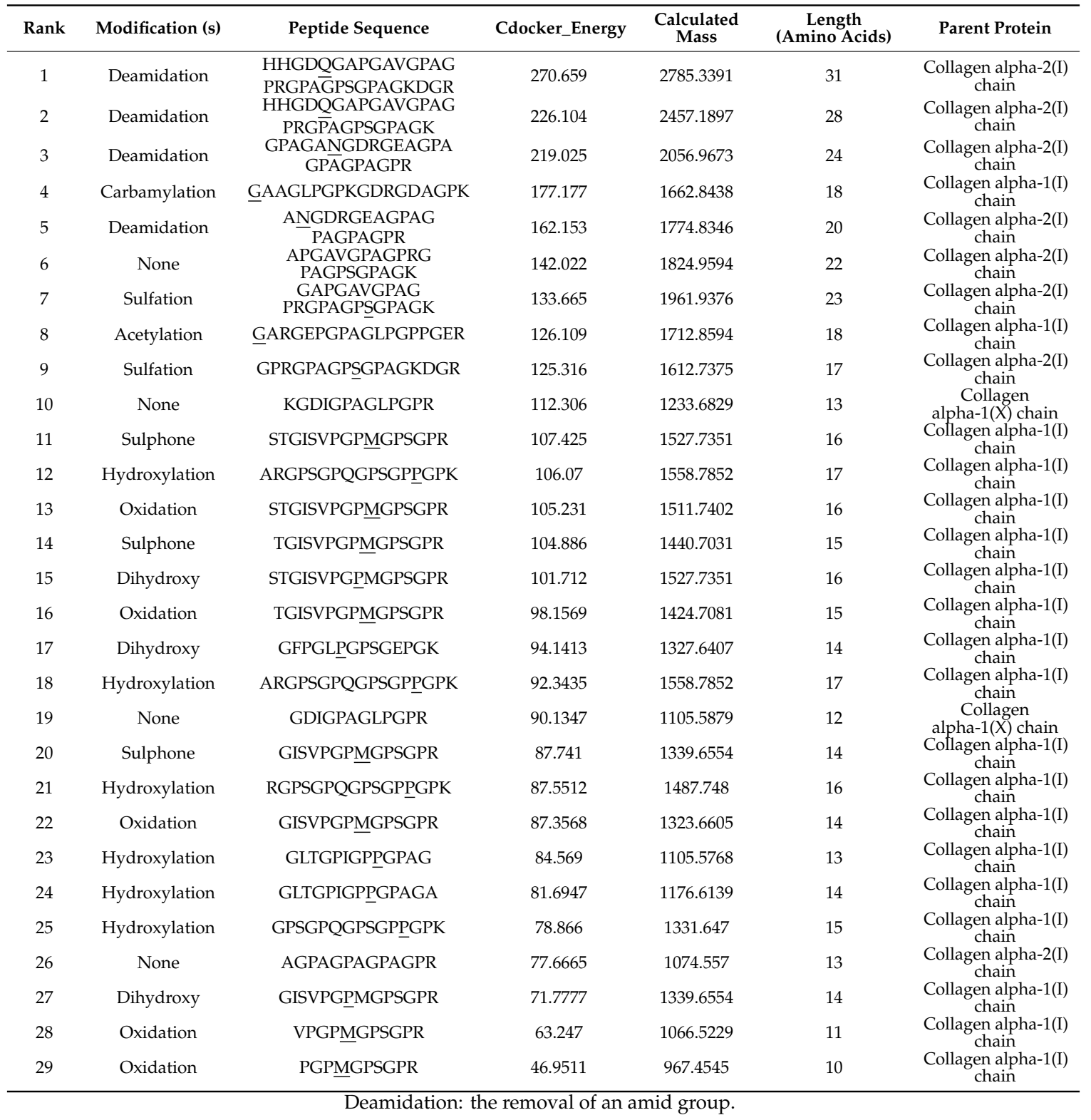

\subsection{Molecular Docking for Screening Peptides that Interact with EGFR}

Prior to docking, a suitable algorithm needed to be selected according to the standard of generated poses and the value of root mean square deviation. We chose to use Libdock and CDOCKER, two classical algorithms in Discovery Studio 2017 software, and MOEdock, an algorithm in Molecular Operating Environment 2014. The principle of Libdock is a scoring function based on hotpot matching, which is able to easily provide a rapid virtual screening $[14,15]$. CDOCKER is an algorithm that uses a CHARMM force field [14]. MOEdock is another forcefield-based scoring function and is able to estimate the free energy of binding with the ligand from a given pose [16]. To select an appropriate algorithm from these three candidates, molecular docking analysis between EGF and EGFR was performed for each of the algorithms. The three algorithms produced four (Libdock), five (CDOCKER), and six (MOEdock) poses, 
respectively. The minimum root mean square deviation values for each of the three algorithms were 15.0634 (Libdock), 14.228 (CDOCKER), and 14.8449 (MOEdock), respectively (Table 2). In addition, the CDOCKER function was the most accurate of the assessed algorithms [13]. Therefore, CDOCKER was the most suitable algorithm for this investigation and was used in subsequent analyses. The "CDOCKER Energy" value (Table 1) indicates the affinity between peptides and EGFR and ranged from 270.659 to 46.9511. Of all analyzed peptides, three peptides had a "CDOCKER Energy" greater than $200 \mathrm{kcal} / \mathrm{mol}$. These three peptides were HHGDQGAPGAVGPAGPRGPAGPSGPAGKDGR (deamidation), HHG DQGAPGAVGPAGPRGPAGPSGPA GK (deamidation), and GPAGANGDRGEAGPAGPAGPAGPR (deamidation). Five peptides shared the same amino acid sequence (GPAGPSGPAGK). Moreover, the only difference between HHGDQGAPGAVGPAGPRGPAGPSGPAGKDGR (deamidation) and HHGDQGAPGAVGPAGPRGPAGPSGPAGK (deamidation) was the amino acid sequence DGR, which was responsible for the minor differences in their "CDOCKER" values. HHGDQGAPGAVGPAGPR GPAGPSGPAGKDGR (deamidation), GPAGANGDRGEAGPAGPAGPAGPR (deamidation), and GP AGPSGPAGKDGR were chosen for further study.

Table 2. Molecular docking between EGF and EGFR using the Libdock, MOEdock, and CDOCKER algorithms.

\begin{tabular}{ccccccccc}
\hline \multicolumn{3}{c}{ Libdock } & \multicolumn{3}{c}{ MOEdock } & \multicolumn{3}{c}{ CDOCKER } \\
\hline Pose & Reference & RMSD $(\AA)$ & Pose & Reference & RMSD $(\AA)$ & Pose & Reference & RMSD $(\AA)$ \\
\hline 1IVO 1 & 1IVO 4 & 15.0634 & 1IVO 1 & 1IVO 6 & 15.2038 & 1IVO 1 & 1IVO 5 & 12.9719 \\
1IVO 2 & 1IVO 4 & 18.8533 & 1IVO 2 & 1IVO 6 & 15.3858 & 1IVO 2 & 1IVO 5 & 14.228 \\
1IVO 3 & 1IVO 4 & 17.3093 & 1IVO 3 & 1IVO 6 & 25.5119 & 1IVO 3 & 1IVO 5 & 21.2551 \\
1IVO 4 & 1IVO 4 & 0 & 1IVO 4 & 1IVO 6 & 14.8449 & 1IVO 4 & 1IVO 5 & 21.8973 \\
- & - & - & 1IVO 5 & 1IVO 6 & 24.1174 & 1IVO 5 & 1IVO 5 & 0 \\
- & - & - & 1IVO 6 & 1IVO 6 & 0 & - & - & - \\
\hline
\end{tabular}

RMSD: root mean square deviation; EGF: epidermal growth factor; EGFR: epidermal growth factor receptor.

\subsection{In Vitro Effect of Synthesized Peptides on MC3T3-E1 Cell Proliferation}

The in vitro cell viability response of MC3T3-E1 cells to the synthesized peptides, at $0.05 \mathrm{mg} / \mathrm{mL}$, was evaluated over 24-72 h using MTT and CCK8 assays. Peptide HHGDQGAPGAVGPAGPRGPA GPSGPAGKDGR (Deamidation) and peptide GPAGANGDRGEAGPAGPAGPAGPR (Deamidation) had the greatest influences on osteoblast MC3T3-E1 viability at $48 \mathrm{~h}(p<0.05)$ and a peptide concentration of $0.05 \mathrm{mg} / \mathrm{mL}$, leading to an increase in viability of $21.4 \%$ and $23.7 \%$, respectively (Figure $2 \mathrm{a}, \mathrm{b}$ ). Unfortunately, the cell effect of the GPAGPSGPAGKDGR peptide on cell viability did not significantly differ from that of the control group.

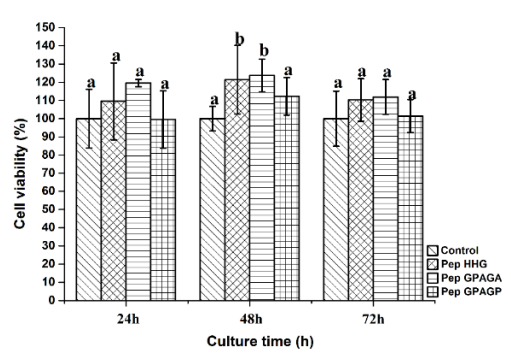

(a)

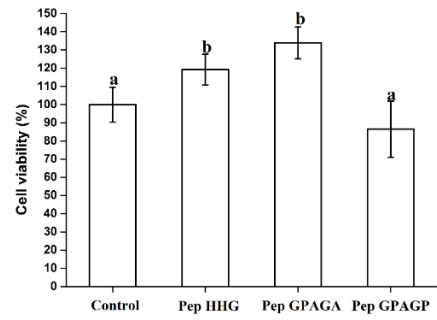

(b)

Figure 2. The relative cell viability of MC3T3-E1 osteoblast cells in response to Pep HHG, Pep GPAGA, and Pep GPAGP was measured using 3-(4,5-Dimethylthiazol-2-yl)-2,5-diphenyltetrazolium bromide (MTT) and CCK8 assays. (a) MTT assay results at 24, 48, and $72 \mathrm{~h}$. (b) CCK8 assay results after $48 \mathrm{~h}$. Different letters represent significant differences between groups, $p<0.05$ Pep HHG: peptide HHGDQGAPGAVGPAGPRGPAGPSGPAGKDGR (Deamidation); Pep GPAGP: peptide GPAGA NGDRGEAGPAGPAGPAGPR (Deamidation); Pep GPAGP: peptide GPAGPSGPAGKDGR. 


\subsection{Effects of Synthesized Peptides on Cell Cycle in MC3T3-E1 Cells}

Peptide HHGDQGAPGAVGPAGPRGPAGPSGPAGKDGR (Deamidation) and peptide GPAGA NGDRGEAGPAGPAGPAGPR (Deamidation), at $0.05 \mathrm{mg} / \mathrm{mL}$, had the greatest effects on osteoblast proliferation. The effect of these peptides on the cell cycle was monitored in MC3T3-E1 cells cultured for 8,16 , and $24 \mathrm{~h}$. Treatment with these peptides resulted in an increase in the percentage of $\mathrm{S}$ phase cells and a reduction in the percentage of G0/G1 phase cells (Figure 3).
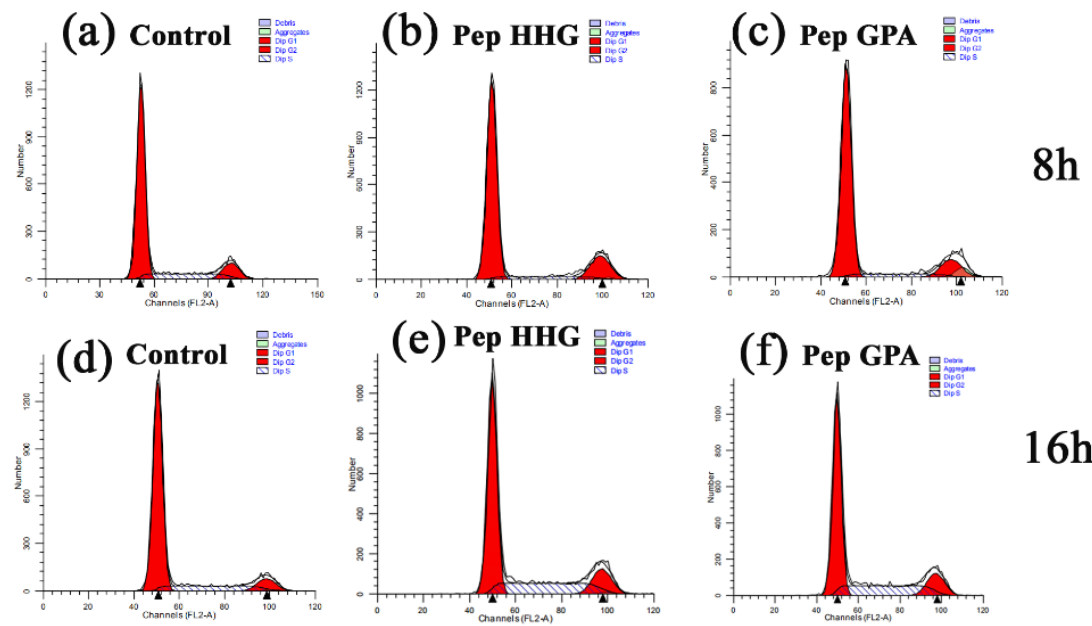

$16 \mathrm{~h}$
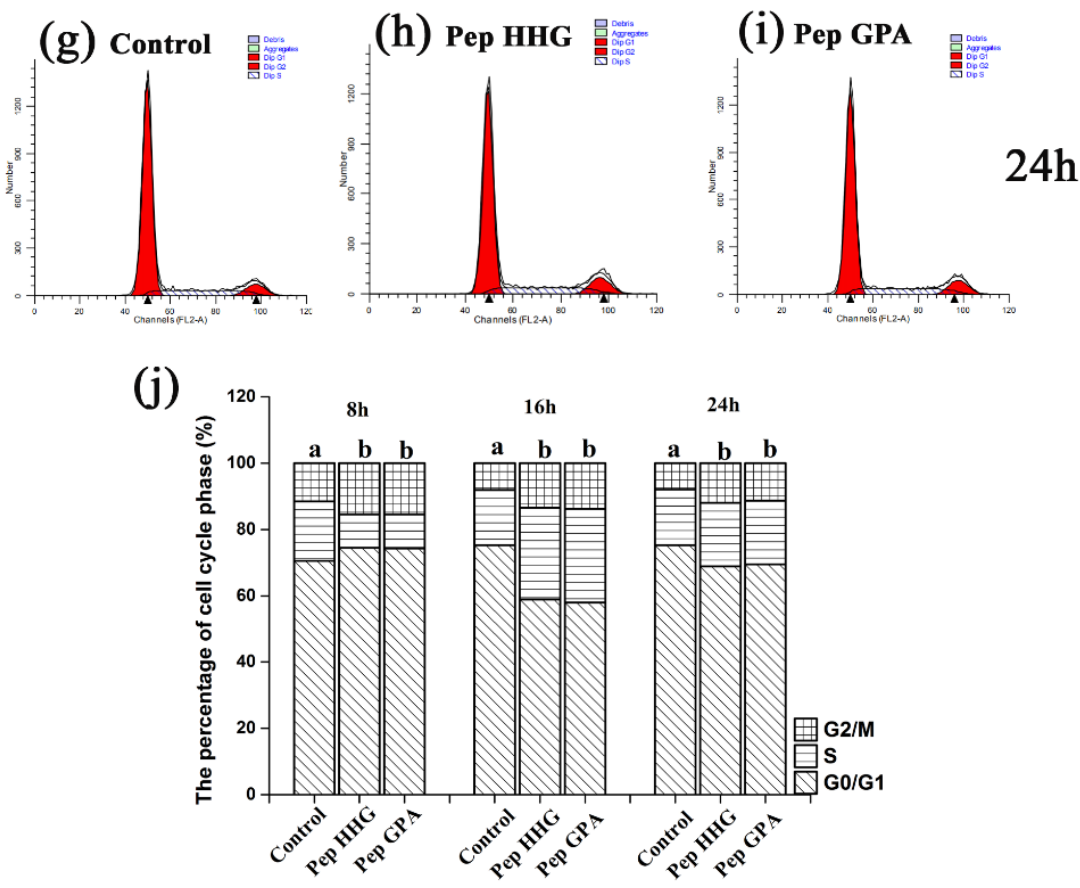

Figure 3. The cell cycle distribution of MC3T3-E1 osteoblasts cells after treatment with Pep HHG and Pep GPA and cultured for varying time periods assessed using flow cytometry (a-i). Cell cycle distribution of MC3T3-E1 osteoblasts in G0/G1, S, or G2/M phases (j). The different letters represent significant difference between groups, $p<0.05$. (a-c) Control MC3T3-E1 osteoblasts and MC3T3-E1 osteoblasts treated with Pep HHG and Pep GPA for $8 \mathrm{~h}$, respectively. (d-f) Control MC3T3-E1 osteoblasts and MC3T3-E1 osteoblasts treated with Pep HHG and Pep GPA for $16 \mathrm{~h}$, respectively. (g-i) Control MC3T3-E1 osteoblasts and MC3T3-E1 osteoblasts treated with Pep HHG and Pep GPA for $24 \mathrm{~h}$, respectively. Pep HHG: peptide HHGDQGAPGAVGPAGPRGPAGPSGPAGKDGR (Deamidation); Pep GPA: peptide GPAGANGDRGEAGPAGPAGPAGPR (Deamidation). 


\subsection{Anti-Apoptotic Effects of the Synthesized Peptides}

The effects of $24 \mathrm{~h}$ treatment with peptide HHGDQGAPGAVGPAGPRGPAGPSGPAGKDGR (Deamidation) and peptide GPAGANGDRGEAGPAGPAGPAGPR (Deamidation) on apoptosis in MC3T3-E1 cells stained with Annexin V (AV)-FITC (Fluoresceine Isothiocyanate) and propidium iodide (PI) were evaluated using flow cytometry. Apoptosis was inhibited by treatment with these two peptides at $0.05 \mathrm{mg} / \mathrm{mL}$ (Figure 4). The total rate of osteoblast MC3T3-E1 apoptosis decreased from 9.845\% to $7.155 \%$ or $3.58 \%$ after treatment with peptide HHGDQGAPGAVGPAGPRGPAGPSGPAGKDGR (Deamidation) and peptide GPAGANGDRGEAGPAGPAGPAGPR (Deamidation), respectively. These results show that peptide GPAGANGDRGEAGPAGPAGPAGPR (Deamidation) had a greater influence on apoptosis than did peptide HHGDQGAPGAVGPAGPRGPAGPSGPAGKDGR (Deamidation). These results also show that these two peptides inhibit early apoptosis in MC3T3-E1 osteoblast cells. The rate of early apoptosis for peptide HHGDQGAPGAVGPAGPRGPAGPSGPAGKDGR (Deamidation) decreased from $9.265 \%$ to $6.205 \%$, and for peptide GPAGANGDRGEAGPAGPAGPAGPR (Deamidation), the rate of early apoptosis decreased from $9.265 \%$ to $3.155 \%$.

(a)

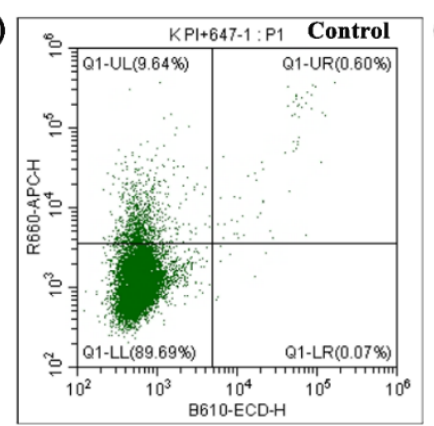

(d)

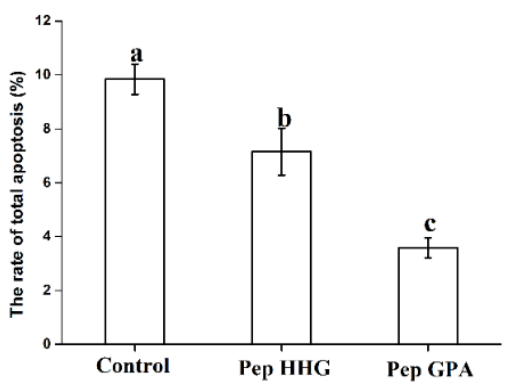

(c)

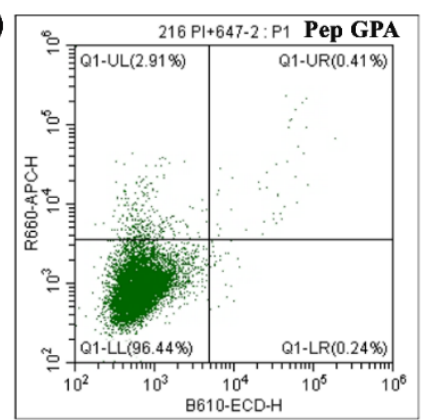

(e)

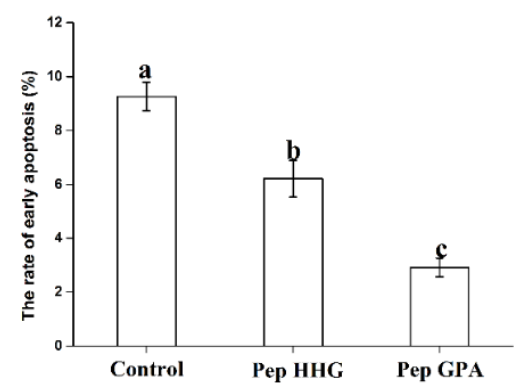

Figure 4. Apoptotic suppression in MC3T3-E1 osteoblast cells lines was measured using flow cytometry $(\mathbf{a}-\mathbf{c})$ and the calculated total apoptotic $(\mathbf{d})$ and early apoptotic (e) rates. The different letters represent significance differences between groups, $p<0.05$. $(\mathbf{a}-\mathbf{c})$ Control cells, cells treated with Pep HHG for $24 \mathrm{~h}$, cells treated with Pep GPA for $24 \mathrm{~h}$, respectively. Pep HHG: peptide HHGDQG APGAVGPAGPRGPAGPSGPAGKDGR (Deamidation); Pep GPA: peptide GPAGANGDRGEAGPA GPAGPAGPR (Deamidation).

\subsection{Molecular Docking between Synthesized Peptides and EGFR}

The HHGDQGAPGAVGPAGPRGPAGPSGPAGKDGR (Deamidation) binding model showed that the peptide interacts with EGFR (Figure 5a-c). The Ser26, Gln384, Glu90, Lys13, and Gly18 receptor side chain formed hydrogen bonds with HHGDQGAPGAVGPAGPRGPAGPSGPAGKDGR (Deamidation). The peptide ligand also hydrophobically interacted with Arg29, His409, Gln408, Leu17, Leu348, Thr15, Phe357, Leu14, Tyr45, and Gln16 in the pocket of the receptor. The GPAGA NGDRGEAGPAGPAGPAGPR (Deamidation) binding model showed that the peptide interacts with EGFR (Figure $5 \mathrm{~d}-\mathrm{f}$ ). The Thr15 and Lys13 side chain in the receptor binding pocket formed hydrogen 
bonds with this peptide. The peptide ligand also hydrophobically interacted with Gln16, Gly18, Leu17, Asn12, His409, Ser11, Leu325, Leu14, and Tyr45 in the pocket of the receptor.

(a)
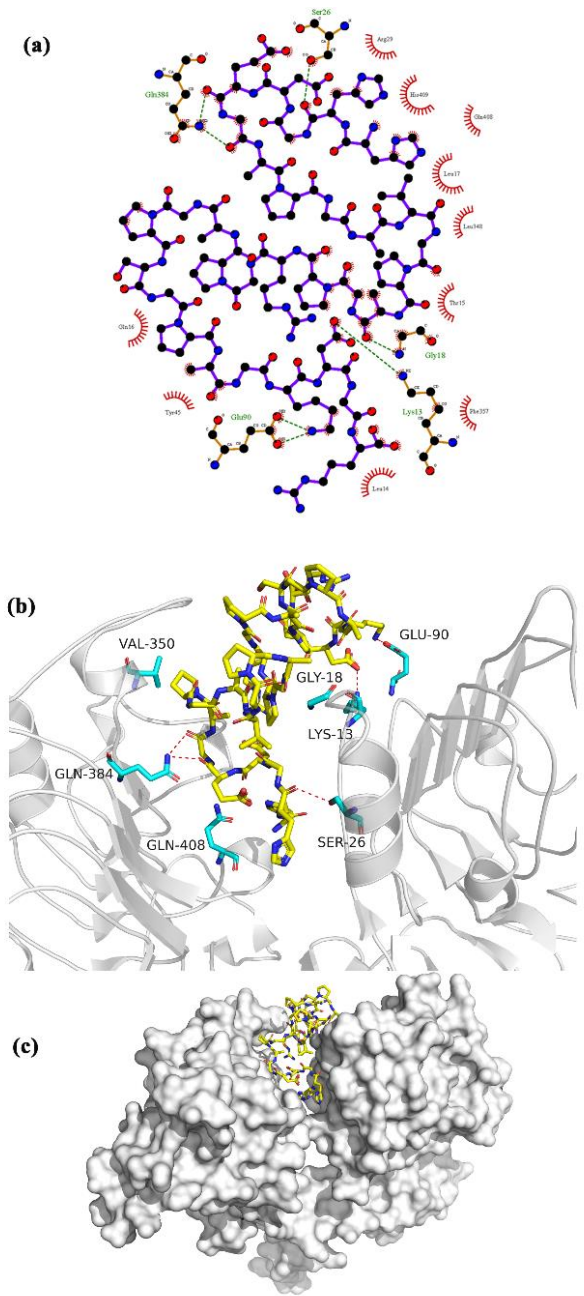

(d)

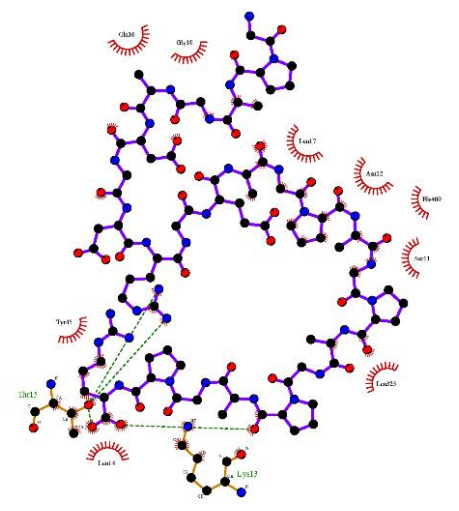

(e)

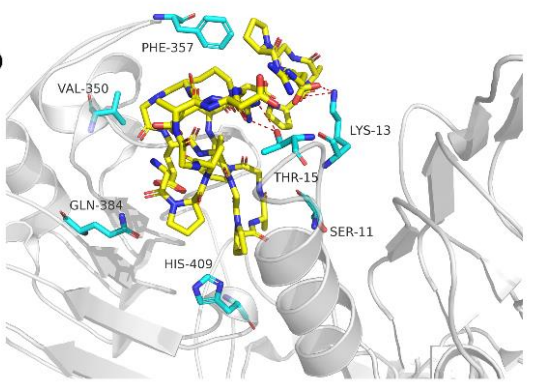

(f)

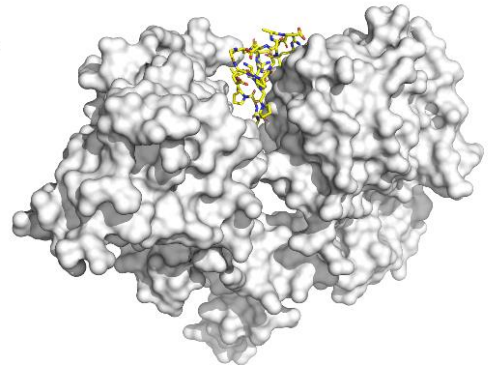

Figure 5. Molecular docking analysis between epidermal growth factor receptor (EGFR) (PDB: 1lVO) and Pep HHG and Pep GPA. (a) The 2D view of the Pep HHG binding model with EGFR. The peptide is shown in ball-stick mode in the middle of the figure. The green dotted lines depict the hydrogen bond with the EGFR amino acid sidechain. The red semicircle line represents the hydrophobic interaction between EGFR amino acid residues and the ligand peptide. (b) The 3D view of the peptide HHG binding model with the target. The carbon atoms of the ligand are in yellow, and the surrounding binding pocket residues are cyan. The receptor backbone is depicted as a light gray ribbon. The red dotted line shows the hydrogen bond between the ligand and receptor. (c) The surface overview of the peptide HHG binding model of the target. The carbon atoms of the ligand are in yellow, and the receptor surface is white. (d) The 2D view of the Pep GPA binding model with EGFR. The peptide is shown in ball-stick mode in the middle of the figure. The green dotted lines depict the hydrogen bond with the EGFR amino acid sidechain. The red semicircle line represents the hydrophobic interaction between EGFR amino acid residues and the ligand peptide. (e) The 3D view of peptide GPA binding model with EGFR. The carbon atoms of the ligand are in yellow, and the surrounding binding pocket residues are cyan. The receptor backbone is depicted as a light gray ribbon. The red dotted line shows the hydrogen bond between the ligand and receptor. (f) The surface overview of the peptide GPA and EGFR binding model. The carbon atoms of the ligand are in yellow, and the receptor surface is white. Pep HHG: peptide HHGDQGAPGAVGPAGPRGPAGPSGPAGKDGR (Deamidation); Pep GPA: peptide GPAGANGDRGEAGPAGPAGPAGPR (Deamidation). 


\section{Discussion}

In recent years, a variety of bioactivities for collagen peptides have been reported, such as antihypertensive [17], antioxidant [18], and antidiabetic actions [19], in addition to beneficial effects on bone. A great volume of evidence indicates that the bioactivity of collagen peptides is related to their molecular weight. For instance, squid skin collagen peptides with the lowest molecular weight (HSSG-III < $2000 \mathrm{Da}$ ) possess the most potent anti-ACE (angiotensin I-converting enzyme) bioactivity among the three fractions, namely, HSSG-I (6-10 kDa), HSSG-II (2-6 kDa), and HSSG-III (<2 Ka) [20]. Dasidicus gigas-derived collagen hydrolysates of low molecular weights, ranging from 500 to $1400 \mathrm{Da}$, show cytotoxic and antiproliferative activities in MCF-7 (human breast carcinoma) and U87 cell lines (glioma) [21]. Collagen peptides from squid skin that are between $1400 \mathrm{Da}$ and $500 \mathrm{Da}$ exert the highest antioxidant ability compared with those of greater mass [20]. Peptides from collagen hydrolysates of cobia skin that are below 700 Da significantly affect the antioxidant properties of these hydrolysates [22]. Two novel peptides with the highest calcium-chelating activity were isolated from pacific cod skincollagen hydrolysates and identified as GDKGESGEAGER and GEKGEGGHR [23]. Similarly, the beneficial effects of collagen on bone health are related to the molecular weight distribution. For example, pigskin collagen with molecular mass below 3000 Da was reported to inhibit bone loss caused by ovariectomizing and improve the microstructure of lumbar vertebrae [24]. Of all fractions, the lowest molecular mass collagen peptides from Crucian skin could bind more calcium and possessed a greater ability to improve calcium bioavailability in the retinoic acid-induced bone loss model [25]. In the present study, BBCH-I, with a molecular size of less than $3000 \mathrm{Da}$, exhibited stronger osteogenic bioactivity. In addition, this fraction contained numerous low molecular weight peptides (Table 1). Moreover, our previous study demonstrated that the fraction with MW 878 Da showed the most potent osteocalcin content increased activity among the four fractions, namely, MW 1370 Da, MW $2900 \mathrm{Da}$, and MW $7747 \mathrm{Da}[26]$. Therefore, there may exist a correlation between osteogenic activity and the molecular size of peptides.

In addition, the types of collagen appear to correlate with the beneficial effects of hydrolyzed collagen on bone health [27]. Type I collagen hydrolysates might exert more impacts on bone health than do other types of collagen hydrolysates because they are capable of providing the primary building blocks required for renewing bone collagen. This is because type I collagen, which plays a vital role in inducement of osteoblastic differentiation, calcification, and the supply of structure and elasticity for bone, is the primary element of bone [28]. Moreover, there are many osteogenic peptides derived from collagen type I including the novel osteogenic peptides, GPSGPAGKDGRIGQPG and GDRGETGPAGPAGPIGPV [29]. In the present study, the majority of identified peptides (Table 1) were from collagen type I. Therefore, our results support the contention that there is a potential correlation between osteogenic peptides and the parental proteins from which they are derived.

The amino acid compositions and peptide sequences of peptides may affect their bioactivity [30]. Peptides comprised of more hydrophobic amino acid residues may have stronger stimulation potencies on osteoblasts as they have the potential to form a greater number of hydrophobic interactions with EGFR, which has a close relationship with bone formation. Additionally, peptides that have a higher percentage of amino acid residues such as glutamic acid may also have greater potential osteogenic activity because of a greater number of possibilities to form hydrogen bonds with EGFR. These inferences are supported by our data (Figure 5). Our results reveal five hydrogen bonds and ten hydrophobic interactions between peptide HHGDQGAPGAVGPAGPRGPAGPSGPAGKDGR (Deamidation) and the EGFR receptor, and two hydrogen $\bar{b}$ onds and nine hydrophobic interactions between peptide GPAGANGDRGEAGPAGPAGPAGPR (Deamidation) and EGFR. Moreover, it has been suggested that hydrogen bonds and hydrophobic interactions contribute to stimulation potency in osteoblasts MC3T3-E1 cells [29].

Cell proliferation is an important phase of bone formation, and both bone formation and skeletal integrity can be affected by the osteoblast's viability [31]. Moreover, bovine bone collagen peptides with higher levels of osteoblast proliferation stimulation can also promote mineralization, 
the final phase of bone formation [10]. In this study, we found that peptide HHGDQGAPGAVG PAGPRGPAGPSGPAGKDGR (Deamidation) and peptide GPAGANGDRGEAGPAGPAGPAGPR (Deamidation) displayed significant osteoblast proliferation stimulation bioactivity $(p<0.05)$. Therefore, these two peptides might be utilized as functional food for stimulating osteoblast proliferation to exert a beneficial effect on bone formation.

Cell proliferation can be adjusted via cell cycle phase alteration, which causes indirect effects on bone formation, including bone mineralization [32]. Collagen peptides can alter cell cycle distribution in B16F10 melanoma cells [33] and chum salmon skin collagen hydrolysates can alter the cell cycle progression [34]. Our results revealed that the proportion of cells in S phase was elevated and the proportion of cells in G0/G1 phase decreased significantly when treated with peptide HHGD QGAPGAVGPAGPRGPAGPSGPAGKDGR (Deamidation) and peptide GPAGANGDRGEAGPAG PAGPAGPR (Deamidation). These results suggest that these peptides can promote the proliferation of MC3T3-E1 osteoblast cells though alteration of cell cycle progression.

Apoptosis, a process that helps organisms remove unwanted cells to precisely control organ function and development, determines osteoblast lifespan in their final phase of formation, which is crucial for bone remodeling [35]. The antiapoptotic effects of collagen hydrolysates from chum salmon skin have been verified [34]. In this study, we found that peptide HHGDQGAPGAVGPAGPRGPA GPSGPAGKDGR (Deamidation) and peptide GPAGANGDRGEAGPAGPAGPAGPR (Deamidation) decreased apoptosis. These results indicate that these peptides might promote proliferation of MC3T3E1 cells by preventing apoptosis.

The EGFR family includes four members: EGFR, erbB2, erbB3, and erbB4. These receptors can be activated via forming oligomers with ligands such as EGF. EGFR, a transmembrane glycoprotein, is frequently activated. The activation of the EGFR signaling pathway is an indicator of cell proliferation and inhibition of apoptosis [36,37]. In other words, the intro cellular signaling cascade, including cell proliferation survival and anti-apoptosis, occurs in response to ligand stimulation, namely, the EGFR signaling pathway is ligand-dependent [38]. In order to screen bioactive peptides from collagen hydrolysates, molecular analysis between EGFR with identified peptides is an efficient and economical way. In addition, molecular docking, a computational simulation method that is able to assist in predicting sites between receptor and ligand, has been used to better screen bioactive peptides with osteoblasts proliferation stimulation from protein hydrolysates [39]. For instance, Shi has successfully screened a peptide, namely ENLPEKADRDQYEL, with promoting proliferation proliferation of osteoblast activity using molecular docking analysis between EGFR and identified peptides from bovine lactoferrin hydrolysate [5]. In this study, peptide HHGDQGAPGAVGPAGPRGPAGPSGPAGKDGR (Deamidation) and peptide GPAGANGDRGEAGPAGPAGPAGPR (Deamidation) may activate EGFR signaling similarly to EGF because they interact with similar sites within the receptor, such as Phe 357 and Val 350. These results suggest that the underlying mechanisms of cell cycle alteration, promotion of cell proliferation, and prevention of apoptosis might be related to the EGFR signaling pathway. However, the mechanisms of the bioactivities observed for the proteins examined need to be further investigated and verified.

\section{Materials and Methods}

\subsection{Materials}

Bovine bone collagen hydrolysates (BBCHs) were prepared in our laboratory. Briefly, collagen was initially extracted from bovine bone, followed by enzymatic hydrolysis using papain and trysin in sequence to produce collagen hydrolysates. MC3T3-E1 osteoblasts and their growth media (Alpha modified Eagle's minimum essential medium (with nucleosides), $\alpha$-MEM-N medium) and media supplement (fetal bovine serum, FBS) were bought from the National Infrastructure Cell line Resource (Beijing, China). The 3-(4,5-Dimethylthiazol-2-yl)-2,5-diphenyltetrazolium bromide (MTT) and CCK8 
kit were purchased from Solarbio Science and Technology Co., Ltd. (Beijing, China). All reagents used were of analytical grade.

\section{2. $B B C H$ Fractionation via Ultrafiltration}

Using a Pellicon ultrafiltration system (Millipore, MA, USA) coupled with a 3000 Da molecular weight (MW) cut-off membrane, $\mathrm{BBCH}$ dissolved in distilled water was fractioned into two fractions: BBCH-1 (MW < $3000 \mathrm{Da}$ ) and BBCH-2 (MW > $3000 \mathrm{Da}$ ). The two fractions were then lyophilized and subjected to cell proliferation assay.

\section{3. $B B C H-1$ and $B B C H-2$ Cell Proliferation Assays}

MC3T3-E1 osteoblasts were seeded in a 96-well plate at a density of 750 cells/well in $\alpha$-MEM-N medium containing 20\% FBS. Cell proliferation assays were performed using an IncuCyte live cell analysis system (Essen Instruments, Ann Arbor, MI, USA). Confluence measurements were used to assess the proliferation stimulation bioactivity of BBCH-1 and BBCH-2 (at concentrations of $0.2,0.1$, and $0.05 \mathrm{mg} / \mathrm{mL}$ ) in osteoblasts.

\subsection{BBCH-1 Nano-HPLC-MS-MS Analysis}

The fraction with the greatest bioactivity (osteoblasts proliferation activity) was dissolved in $10 \mu \mathrm{L}$ distilled water containing $0.1 \%$ formic acid. Subsequently, the fraction was further purified using a nano Aquity UPLC system (Waters, Milford, MA, USA) and analyzed using quardrupole-Orbitrtrap mass spectrometry (Q-Exactive; Thermal Fisher Scientific, Bremen, Germany) coupled with an online nano-electrospray ion source. The sample of $4 \mu \mathrm{L}$ was loaded onto the trap column (Thermal Scientific Acclaim PepMap C18, $100 \mu \mathrm{m} \times 2 \mathrm{~cm}$ ) with a flow rate of $10 \mu \mathrm{L} / \mathrm{min}$ for $3 \mathrm{~min}$ and then purified onto the analytical column (Acclaim PepMap C18, $75 \mu \mathrm{m} \times 25 \mathrm{~cm}$ ) using a linear gradient $(5 \% \mathrm{~B}-30 \% \mathrm{~B}$ in $85 \mathrm{~min}, \mathrm{~A}$ : distilled water containing $0.1 \%$ of formic acid; B: Acetonitrile containing $0.1 \%$ of formic acid) at a flow rate of $300 \mathrm{~nL} / \mathrm{min}$ with a constant column temperature of $45^{\circ} \mathrm{C}$. Electrospray voltage of 2.0 KV versus the inlet of the mass spectrometer was used. All MS/MS data were obtained through HPLC-MS_MS analysis and then searched against the Uniprot proteome Bos taurus database via PEAKS Studio 6.0 software (Bioinformatics Solution Inc., Waterloo, ON, Canada).

\subsection{Molecular Docking}

Molecular docking analysis between peptides with greater bioactivity and EGFR was performed using Discovery Studio (DS) software and Molecular Operating Environment (MOE) software (version 2014, WeiCompu Ltd., Beijing, China). The EGFR-EGF protein-ligand complex (PDB ID: 1IVO) crystal structure was obtained from the RCSB Protein Data Bank (https://www.rcsb.org) and served as the docking target and the template for comparing the docking mode peptides bound to EGFR [40]. CDOCKER, Libdock, and Moedock classical algorithms were used for the docking model. A suitable algorithm needed to be validated based on the standard of the root mean square deviation (RMSD) values and the number of generated poses. For receptor preparation in the DS software, removing water molecules, adding hydrogen, removing EGF ligands, and constructing the missing loop regions were performed by the Prepare Protein module. Additionally, the original position (EGF site) in the $1 \mathrm{IVO}$ complex served as the active site for docking simulation. For receptor prepared in the MOE software, the protonation and orientation of the hydrogens were optimized by LigX.

\subsection{Peptide Synthesis}

Peptides with greater osteogenic activity were synthesized using the solid-phase method (GL Biochem Ltd., Shanghai, China) based on the results of molecular docking analyses. These peptides were $96 \%$ pure and their sequences was validated using HPLC-MS-MS. The remaining $4 \%$ was TFA (tallow fatty acid) salt and some by products. 


\subsection{MTT Assay and CCK8 Assay for Synthesized Peptides}

Cell medium containing 750 MC3T3-E1 cells $(200 \mu \mathrm{L})$ was seeded into 96-well plates and cultured in a Stericycle $\mathrm{CO}_{2}$ incubator (Thermal fisher Scientific Inc., Waltham, MA, USA). After incubating for 24,48 , and $72 \mathrm{~h}$, proliferation assays were performed using the MTT and CCK8 methods to evaluate the bioactivity of the synthesized peptides in stimulating osteoblast proliferation.

\subsection{Cell Cycle Analysis}

MC3T3-E1 osteoblast cells were seeded into six-well plates at a density of $3 \times 10^{5}$ cells/well and treated with $\alpha$-MEM-N medium containing synthesized peptides $(0.05 \mathrm{mg} / \mathrm{mL})$ for 8,16 , and $24 \mathrm{~h}$. Cells not treated with peptides were used as the control. Harvested cells were subjected to cell cycle analysis using flow cytometry (FACS Calibur, Becton Dickson, San Jose, CA, USA).

\subsection{Apoptosis Analysis}

MC3T3-E1 cells seeded into six-well plates at a density of $3 \times 10^{5}$ cells/well, either treated with synthesized peptides or untreated, were cultured for $24 \mathrm{~h}$. Then, the FITC-annexin V/PI detection kit was used to analyze apoptosis.

\subsection{Statistical Analysis}

The statistically significant differences were calculated using SPSS software (version 20.0, SPSS Inc., Chicago, IL, USA). Significant differences between the mean values of three or more experiments were calculated using one-way analysis of variance. With LSD (L) (least-significant-difference) and S-N-K (Studen-Newman-Keuls) procedure, $p<0.05$ was considered statistically significant.

\section{Conclusions}

Two novel peptides, HHGDQGAPGAVGPAGPRGPAGPSGPAGKDGR (Deamidation) and GPA GANGDRGEAGPAGPAGPAGPR (Deamidation), were identified based on their affinity with the EGFR receptor and their bioactivities. Additionally, our results show that these identified bioactivity peptides from bovine bone collagen hydrolysates function through the underlying mechanisms of anti-apoptosis, cell cycle alteration, and stimulation of proliferation, and might be related to the EGFR signaling pathway. Moreover, there might be a correlation between the bioactivities of peptides and their sequences, molecular sizes, and the types of collagen from which they are derived. Our results provide a foundation for the application of these peptides as functional components of functional gelatin products.

Author Contributions: Conceptualization, J.W. and Y.G.; investigation, J.W.; writing-original draft preparation, J.W.; writing-review and editing, J.L. All authors have read and agreed to the published version of the manuscript.

Funding: This research received no external funding.

Conflicts of Interest: The authors declare no conflict of interest.

\section{References}

1. Chim, S.M.; Tickner, J.; Chow, S.T.; Kuek, V.; Guo, B.; Zhang, G.; Rosen, V.; Erber, W.; Xu, J. Angiogennic factors in bone local environment. Cytokine Growth Factor Rev. 2013, 24, 297-310. [CrossRef] [PubMed]

2. Aasetha, J.; Boivinbc, G.; Andersen, O. Osteoporosis and trace elements. J. Trace. Elem. Med. Biol. 2012, 26, 149-152. [CrossRef] [PubMed]

3. $\quad$ Pereira, A.G.; Chiba, F.Y.; de Lima, C.; Mattera, M.S.; Pereira, R.F.; de Cássia Alves Nunes, R.; Tsosura, T.V.S.; Okamoto, R.; Sumida, D.H. Effects of fluoride on insulin signaling and bone metabolism in ovariectomized rats. J. Trace. Elem. Med. Biol. 2017, 39, 140-146. [CrossRef] [PubMed]

4. Eriksen, E.F.; Díez-Pérez, A.; Boonen, S. Update on long-term treatment with bisphosphoanates for postmenopausal osteoporosis: A systematic review. Bone 2014, 58, 126-135. [CrossRef] 
5. Shi, P.; Liu, M.; Fan, F.J.; Chen, H.; Yu, C.P.; Lu, W.H.; Du, M. Identification and mechanism of peptides with activity promoting osteoblast proliferation from bovine and lactoferrin. Food Biosci. 2018, 22, $19-25$. [CrossRef]

6. Park, S.H.; Song, T.J.; Bae, T.S.; Khang, G.; Choi, B.H.; Park, S.R.; Min, B.H. Comparative analysis of collagens extracted from different animal sources for application of cartilage tissue engineering. Int. J. Precis. Eng. Man. 2012, 13, 2059-2066. [CrossRef]

7. Tziveleka, L.A.; Loannou, E.; Tsiourvas, D.; Berillis, P.; Foufa, E.; Roussis, V. Collagen from the marine sponges Axinella cannabina and Suberites carnosus: Isolation and morphological, biochemical, and biophysical characterization. Mar. Drugs 2017, 15, 152. [CrossRef]

8. Moskowitz, R.W. Role of collagen hydrolysate in bone and joint disease. Semin. Arthritis Rheum. 2000, 30, 87-99. [CrossRef]

9. De Almeida Jackix, E.; Cúneo, F.; Amaya-Farfan, J.; de Assunção, J.V.; Quintaes, K.D. A food supplement of hydrolyzed collagen improves compositional and biodynamic characteristics of vertebrae in ovariectomized rats. J. Med. Food 2010, 13, 1385-1390. [CrossRef]

10. Liu, J.L.; Zhang, B.; Song, S.J.; Ma, M.; Si, S.Y.; Wang, Y.H.; Xu, B.X.; Feng, K.; Wu, J.G.; Guo, Y.C. Bovine collagen peptides compounds promote the proliferation and differentiation of MC3T3-E1 pre-osteoblasts. PLoS ONE. 2014, 4, e99920. [CrossRef]

11. Liu, J.L.; Song, S.J.; Si, S.Y.; GAO, J.; Wang, Y.H.; Qin, Y.Y.; Chen, X.N.; Guo, Y.C. Effect of collagen peptide and calcium citrate on bone loss in ovariectomized rats. Chin. J. Osteoporos. Bone Miner. Res. 2015, 8, 334-339.

12. Wang, Y.L.; Liu, J.L.; Li, B.C.; Wang, Y.H.; Yang, G.A.; Gao, L.M.; Gengen, T.; Wang, F.R.; Guo, Y.C. Effects of collagen peptide combined with Caltrate $\mathrm{D}$ on the treatment of postmenopausal osteoporosis. Chin. J. Osteoporos. Bone Miner. Res. 2019, 25, 228-232.

13. Xie, M.; Dart, D.A.; Guo, T.; Xing, X.F.; Ji, J.F. MicroRNA-1 acts as a tumor suppressor microRNA by inhibiting angiogenesis-related growth factors in human gastric cancer. Gastric Cancer 2017, 21, 41-54. [CrossRef] [PubMed]

14. Rao, S.N.; Head, M.S.; Kulkarni, A.; LaLonde, J.M. Validation studies of the site-directed docking program libdock. J. Chem. Inf. Mode 2007, 47, 2159-2171. [CrossRef]

15. Kang, D.; Pang, X.C.; Lian, W.W.; Xu, L.J.; Wang, J.H.; Jia, H.; Zhang, B.Y.; Du, G.H. Discovery of VEGFR2 inhibitors by integrating naï ve Bayesian classification, molecular docking and drug screening approaches. RSC Adv. 2018, 8, 5286-5297. [CrossRef]

16. Wadood, A.; Riaz, M.; Jamal, S.B.; Shah, M.; Lodhi, M.A. Molecular docking study of p4-benzoxaborolesubstituted ligands as inhibitors of hcv ns3/4a protease. Bioinformation 2013, 9, 309-314. [CrossRef]

17. Fu, Y.; Young, J.F.; Løkke, M.M.; Lametsch, R.; Aluko, R.E.; Therkildsen, M. Revalorisation of bovine collagen as a potential precursor of angiotensin i-converting enzyme (ace) inhibitory peptides based on in silico and in vitro protein digestions. J. Funct. Foods 2016, 24, 196-206. [CrossRef]

18. Cai, L.; Wu, X.; Zhang, Y.; Li, X.; Ma, S.; Li, J. Purification and characterization of three antioxidant peptides from protein hydrolysate of grass carp (ctenopharyngodon idella) skin. J. Funct. Foods 2015, 16, 234-242. [CrossRef]

19. Zhang, Y.; Chen, R.; Chen, X.; Zeng, Z.; Ma, H.; Chen, S.W. Dipeptidyl peptidase iv-inhibitory peptides derived from silver carp (hypophthalmichthys molitrix) proteins. J. Agric. Food Chem. 2016, 64, 831-839. [CrossRef]

20. Lin, L.; Lv, S.; Li, B. Angiotensin-I-converting enzyme (ACE)-inhibitory and antihypertensive properties of squid skin gelatin hydrolysates. Food Chem. 2012, 131, 225-230. [CrossRef]

21. Alemán, A.; Pérez-Santin, E.; Bordenave-Juchereau, S.; Arnaudin, I.; Gómez-Guillén, M.C.; Montero, P. Squid gelatin hydrolysates with antihypertensive, anticancer and antioxidant activity. Food Res. Int. 2011, 44, 1044-1051. [CrossRef]

22. Yang, J.I.; Ho, H.Y.; Chu, Y.J.; Chow, C.J. Characteristic and antioxidant activity of retorted gelatin hydrolysates from cobia (Rachycentron canadum) skin. Food Chem. 2008, 110, 128-136. [CrossRef] [PubMed]

23. Wu, W.; Li, B.; Hou, H.; Zhang, H.; Zhao, X. Isolation and identification calcium-chelating peptides from pacific cod skin gelatin and their binding properties with calcium. Food Funct. 2017, 8, 4441-4448. [CrossRef] [PubMed] 
24. Kim, H.K.; Kim, M.G.; Leem, K.H. Osteogenic activity of collagen peptide via ERK/MAPK pathway mediated boosting of collagen synthesis and its therapeutic efficacy in osteoporotic bone by back-scattered electron imaging and microarchitecture analysis. Molecules 2013, 18, 15474-15489. [CrossRef] [PubMed]

25. Liu, J.L.; Si, S.Y.; Qin, Y.Y.; Zhang, B.; Song, S.J.; Guo, Y.C. The effect of different molecular weight collagen peptides on mc3t3-e1 cells differentiation. Bio. Med. Mater. Eng. 2015, 26, S2041-S2047. [CrossRef] [PubMed]

26. Hou, T.; Liu, Y.; Guo, D.; Li, B.; He, H. Collagen peptides from crucian skin improve bioavailability and structural characterization by HPLC-ESI-MS/MS. J. Agric Food Chem. 2017, 65, 8847-8854. [CrossRef] [PubMed]

27. León-López, A.; Morales-Peñaloza, A.; Martínez-Juárez, V.M.; Vargas-Torres, A.; Zeugolis, D.I.; Aguirre-Álvarez, G. Hydrolyzed Collagen-Sources and Applications. Molecules 2019, 24, 4031. [CrossRef]

28. Nomura, Y.; Oohashi, K.; Watanabe, M.; Kasugai, S. Increase in bone mineral density through oral administration of shark gelatin to ovariectomized rats. Nutrition 2005, 21, 1120-1126. [CrossRef]

29. Ye, M.L.; Jia, W.; Zhang, C.H.; Shen, Q.S.; Zhu, L.Y.; Wang, L.S. Preparation, identification and molecular docking study of novel osteoblast proliferation-promoting peptides from Yak (Bos grunniens) bones. RSC Adv. 2019, 9, 14627-14637. [CrossRef]

30. Meisel, H.; FitzGerald, R.J. Biofuntional peptides from milk proteins: Mineral binding and cytomodulatory effects. Curr. Pharm. Des. 2003, 9, 1289-1295.

31. Manolagas, S.C. Birth and death of bone cells: Basic regulatory mechanisms and implications for the pathogenesis and treatment of osteoporosis. Endocr. Rev. 2000, 21, 115-137. [PubMed]

32. Drissi, H.; Hushka, D.; Aslam, F.; Nguyen, Q.; Buffone, E.; Koff, A.; van Wijnen, A.; Lian, J.B.; Stein, J.L.; Stein, G.S. The cell cycle regulator p27kip1 contributes to growth and differentiation of osteoblasts. Cancer Res. 1999, 59, 3705-3711. [PubMed]

33. Castro, G.A.; Maria, D.A.; Bouhallab, S.; Sgarbieri, V.C. In vitro impact of a whey protein isolate (WPI) and collagen hydrolysates on B16F10 melanoma cells proliferation. J. Dermatol Sci. 2009, 56, 51-57. [CrossRef] [PubMed]

34. Fu, Y.; Zhao, X.H. In vitro responses of hFOB1.19 cells towards chum salmon (Oncorhynchus keta) skin gelatin hydrolysates in cell proliferation, cycle progression and apoptosis. J. Funct. Foods 2013, 5, 279-288. [CrossRef]

35. Xing, L.; Boyce, B.F. Regulation of apoptosis in osteoclasts and osteoblastic cells. Biochem. Biophys. Res. Commun. 2005, 328, 709-720. [CrossRef]

36. Burgess, A.W. EGFR family: Structure physiology signalling and therapeutic targets. Growth Factors 2008, 26, 263-274. [CrossRef]

37. Martinelli, E.; Ciardiello, D.; Martini, T.; Troiani, T.; Cardone, C.; Vitiello, P.P.; Normanno, N.; Rachiglio, A.M.; Maiello, E.; Latiano, T.; et al. Implementing anti-epidermal growth factor receptor (EGFR) therapy in metastatic colorectal cancer: Challenges and future perspectives. Ann. Oncol. 2020, 31, 30-40. [CrossRef]

38. Schlessinger, J. Receptor tyrosine kinases: Legacy of the first two decades. Cold Spring Harb. Perspect. Biol. 2014, 6, a008912. [CrossRef]

39. Pripp, A.H.; Ardoe, Y. Modelling relationship between angiotensin-(I)-converting enzyme inhibition and the bitter taste of peptides. Food Chem. 2007, 102, 880-888. [CrossRef]

40. Ogiso, H.; Ishitani, R.; Nureki, O.; Fukai, S.; Yamanaka, M.; Kim, J.H.; Saito, K.; Sakamoto, A.; Inoue, M.; Shirouzu, M.; et al. Crystal structure of the complex of human epidermal growth factor and receptor extracellular domains. Cell 2002, 110, 775-787. [CrossRef]

Sample Availability: Bovine bone collagen hydrolysates were available from the authors. 